\title{
UBE3A, un gène inattendu impliqué dans le syndrome d'Angelman
}

Les syndromes de Prader Willi et d'Angelman sont associés à la région chromosomique 15q11-q13 soumise à empreinte parentale $\left(\mathrm{m} / \mathrm{s} n^{\circ} 2\right.$, vol. 9, p. 232) et explorée de façon intensive depuis plusieurs années. La cartographie physique de cette région fut d'autant plus facile que, dans $70 \%$ des cas environ, la mutation correspond à une délétion interstitielle. Dans une même famille, celle-ci peut indifféremment être responsable de la naissance d'un enfant atteint de syndrome de Prader-Willi si elle est héritée du père, ou d'un enfant atteint de syndrome d'Angelman si elle portée par le chromosome 15 maternel. Ces syndromes apparaissent ainsi de façon «alternative" parce que la délétion est suffisamment étendue pour emporter à la fois la région contenant le(s) gène(s) du syndrome de Prader-Willi et celle, plus distale, du syndrome d'Angelman.

Pour le syndrome de Prader-Willi, les gènes candidats soumis à empreinte et exprimés uniquement dans le chromosome paternel ne manquent pas, le favori étant le gène $S N R P N$ dont le rôle exact reste encore à définir $(\mathrm{m} / \mathrm{s}$ $n^{\circ} 6$, vol. 12, p. 835). En revanche, dans la région du syndrome d'Angelman, la candidature du seul gène localisé dans la plus petite région commune, $U B E 3 A$, n'avait pas été retenue, pour l'excellente raison qu'il échappe à l'empreinte et s'exprime de façon biallélique [1].

Or, deux équipes américaines viennent simultanément de démontrer qu'on avait eu tort de rejeter ce gène et qu'il est bel et bien en cause dans le syndrome d'Angelman [2, 3]. Le premier élément déterminant fut la découverte d'un point de cassure situé dans le gène $U B E 3 A$, chez une fille atteinte d'un syndrome d'Angelman et ayant reçu de sa mère un chromosome 15 porteur d'une inversion paracentrique équilibrée. En l'absence de tout autre remaniement, la rupture du gène UBE3A semblait donc, dans ce cas, responsable de la maladie.

Mais, pour le prouver, encore fallaitil démontrer que ce gène était muté dans les rares cas de syndromes d'Angelman qui n'ont ni délétion, ni disomie uniparentale (avec deux chromosomes 15 paternels), ni anomalie de la méthylation $\left(\mathrm{m} / \mathrm{s} n^{\circ} 12\right.$, vol. 12, p. 1441) et qui doivent donc résulter d'une mutation.

Effectivement, six mutations intragéniques viennent d'être décelées, qui peuvent toutes entraîner une perte de fonction du produit du gène. Comment alors expliquer l'absence d'empreinte à laquelle il était logique de s'attendre dans cette maladie qui n'est transmise que par une lésion du chromosome maternel ?

On savait que l'empreinte parentale d'un gène pouvait être limitée à une espèce [4], à un tissu, à un stade du développement [5] ou dépendre d'un promoteur ou d'un épissage alternatif [6]. On dispose du reste d'un parfait exemple: celui du gène IGF2 (pour insulin growth factor) pour lequel de nombreux mécanismes d'empreinte furent décrits [7].

Mais par quel mécanisme le gène $U B E 3 A$ peut-il entraîner ce sévère retard mental qui est l'élément majeur du syndrome d'Angelman? Le produit du gène $U B E 3 A$ fut d'abord identifié dans un complexe avec la protéine E6, produite par certains papillomavirus, d'où son nom initial E6-AP (pour protéine associée à E6) [8]. Le complexe E6-AP/UBE3A fait partie des ubiquitine-protéines ligases possédant un domaine hect (pour homologue du domaine carboxy-terminal E6-AP). Il est impliqué dans le catabolisme protéique intracellulaire, passant par le transfert d'ubiquitine de la protéine E1 vers les protéines E2 et E3 qui ont des fonctions de reconnaissance et de couplage spécifiques de certains substrats [9].

Chez la drosophile, on savait que la voie protéique de l'ubiquitine jouait un rôle au cours du développement du système nerveux: la mutation Bendless, affectant une enzyme de conjugaison de l'ubiquitine, entraîne en effet des anomalies dans l'orientation des axones et la formation des synapses de certains neurones [9]. De plus, une expression très forte du transcrit $U B E 3 A$ a été observée dans le cerveau foetal.

Il est donc possible que l'absence, ou les mutations de $U B E 3 A$, entraîne un défaut dans la dégradation d'une protéine au cours du développement cérébral. Pour aller encore plus avant dans les hypothèses, le gène maternel pourrait jouer un rôle particulier dans certains tissus et à un moment donné du développement. La même mutation sur le gène paternel pourrait donner une anomalie récessive, sans retentissement sur le phénotype. Si elle existait à l'état homozygote, elle devrait produire un phénotype différent du syndrome d'Angelman. (observation hautement improbable en pathologie humaine, mais éventuellement réalisable sur un modèle animal par invalidation du gène et transmission à la descendance).

La découverte de l'implication d'UBE3A, dans le syndrome d'Angelman est inattendue et importante à 
bien des égards. Elle démontre que l'empreinte parentale chez l'homme peut encore nous réserver des surprises et révèle le rôle encore peu connu, mais probablement plein d'avenir, de l'ubiquitinylation dans le développement cérébral et les fonctions neurologiques chez l'homme.

S.G.

1. Nakao M, Sutcliffe JS, Durtschi B, Mutirangura $\mathrm{A}$, Ledbetter $\mathrm{DH}$, et al. Imprinting analysis of three genes in the Prader-Willi/Angelman region: SNRPN, E6-associated protein, and
PAR-2 (D15S225E). Hum Mol Genet 1994; 3 : 309-15.

2. Kishino T, Lalande M, Wagstaff J. UBE3A/E6AP mutations cause Angelman syndrome. Nature Genet 1997; 15: 70-3.

3. Matsuura T, Sutcliffe JS, Fang P, Galjaard RJ, Jiang YH Benton CS, et al. De novo truncating mutations in E6-AP ubiquitin-protein ligase gene (UBE3A) in Angelman syndrome. Nature Genet 1997; 15: 74-7.

4. DeChiara TM, Robertson EJ, Efstrafiadis A. Parental imprinting of the mouse insulin-like growth factor II gene. Cell 1991; 64: 849-59.

5. Deltour L, Montagutelli X, Guenet JL, Jami J, Paldi A. tissue- and development stage specific imprinting of the mouse proinsulin gene Ins2. Dev Biol 1995; 168: 686-8.

6. Vu TH, Hoffman AR. Promotor specific imprin- ting of the human insulin-like growth factor II gene. Nature 1994; 371 : 714-7.

7. Dandolo L. Empreinte parentale des gènes H19 et IGF-2. Med Sci 1995; 11 : 1483-6.

8. Huibregtse JM, Scheffner M, Howley PM. Cloning and expression of the cDNA for E6-AP, a protein that mediates the interaction of the papillomavirus E8 oncoprotein with p53. Mol Cell Biol 1993; 13: 775-84.

9. Carillo S, Pariat M, Jariel-Encontre I, Steff A Piechaczyk M. Le catabolisme protéique intracellulaire: une fonction biologique majeure. Partie I : les mécanismes de dégradation. Med Sci $1995 ; 11: 723-34$.

10. Oh CE, McMahon R, Benzer S, Tanouye MA. Bendless, a drosophila gene affecting neuronal connectivity encodes a ubiquin-conjugating enzyme homolog. J Neurosci 1994; 14 : 3166-79.

\section{find II Ingtitut Pnateur}

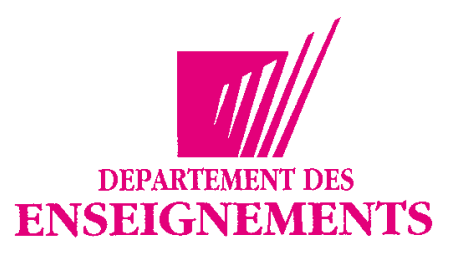

